\title{
Computer Adaptive Testing and Cloud Computing
}

\author{
Joel Suárez-Cansino ${ }^{1}$, Luis R. Morales-Manilla ${ }^{2}$, and Virgilio López-Morales ${ }^{1}$ \\ 1 Autonomous University of Hidalgo State, \\ Institute of Exact Sciences and Engineering, \\ Research Center of Information Technologies and Systems, \\ Mexico \\ 2 Polytechnic University of Tulancingo, Engineering Division, \\ Mexico \\ \{joel.suarez, virgilio.lopez\}@uaeh.edu.mx \\ luisr.morales@upt.edu.mx \\ http://www . uaeh.edu.mx
}

\begin{abstract}
Computer Adaptive Testing (CAT) is an example of a Computer Based Test (CBT) and is one of the main trending topics in the area of knowledge testing and, more recently, in e-learning or in Intelligent Tutoring Systems scenarios. The Item Response Theory (IRT) defines the theoretical basis of a CAT implementation, which assumes the existence of a repository of properly calibrated items that is used during the testing process of a particular examinee. The calibration and adaptation are based on an Item Characteristic Curve (ICC) related to an specific model, being Rasch's models the most widely used. CAT systems require high computational cost to implement the calibration and evaluation processes and the amount of concurrent users at a time could be large enough. Thus, the platform must support high concurrency and availability to perform a desired level of functionality. Technological tendencies in computing offer each time better platforms to develop and manage big collections of data for its processing and relevant information extraction. This paper presents a perspective of using new technologies in CAT as an alternative of implementation. Particularly, the use of a cloud computing platform as current alternative for online CAT systems using the capabilities of multicore processing and big amount of RAM that offers the cloud, to resolve the proper mathematical equations related to psychometric models and the operations described in their algorithms in a real evaluation scheme.
\end{abstract}

Keywords: Computer adaptive testing, item response theory, cloud computing, big data, multicore processing

\section{Introduction}

Computer Adaptive Testing (CAT) is an example of an informatic system oriented to knowledge, skills and behavior explorations, among others questions, 
related to a person in an specific working area. Recently, this kind of systems has become very well known in online e-learning scenarios, as a result of the several benefits that these platforms provide in comparison with those given by Classical Testing Systems (CTS) [8]. CAT systems assume the existence of a pool of perfectly calibrated items, which are used during the testing of an specific person. The calibration process allows to determine the value of the parameters associated to certain psychometric features of the examinees and the items.

The essential idea in CAT systems consists in presenting to a given examinee one item after another, depending on the responses given to previous items along the testing process. If the response is incorrect, then the next item has a lower difficulty than the current one; on the other hand, if the response is correct, then the next item has a higher difficulty. This characteristic makes the entire process adaptable, which means that the item presentation adapts to the examinees' knowledge, in opposition to what classical testing does.

The implementation of this kind of environment is not trivial, since mathematical models and sophisticated algorithms are used for items' calibration and the adapting testing process itself. Particularly, the calibration process requires to find the solutions of a system of non-linear equations, while the adaptive testing computation needs at least to search for the solution of one non-linear function in quasi real time. In addition to this, the users can access the system in a concurrent way, which obviously impacts the system's and the hosting platform's performances.

Currently, there do exist informatics environments where a CAT system can be hosted with the aforementioned features, and they provide services over the Internet. In this sense, technological advances have changed the paradigm of implementing the solution in-house ('on-premises') to implement the solution on the Cloud ('off-premises').

Cloud computing is every day a more often used concept in computer systems. The Cloud-based service providers offer each time more and better management options, as well as benefits related to the accessible cost which depends of the user needs. So that nowadays it is possible that every individual has her/his own personal Cloud, which makes a proper environment for the hosting, creation and maintenance of applications for a wide variety of topics such as, for example, the automatized testing systems [8]. Cloud computing is an information service that offers software, platforms and infrastructure to an organization. Cloud computing technology incorporates different types of private, community, public and hybrid Clouds [8].

In the technology industry, Cloud computing is exponentially changing the implementation of information technology services. This is due to the fact that Cloud computing is a new information technology platform, that will positively change the nature of information management systems in the organizations.

The Cloud, which is another name for Cloud computing, is sometimes referred to as utility computing, since it uses interconnects networked devices to share information resources [12]. The online software and virtual maintenance of Internet infrastructure are among the benefits of Cloud computing for organizations, 
and they can be synchronized from any geographic location [1].

Cloud computing uses the power of large computing devices that work on a common software format making parallel networks possible [10]. The large processing power of Cloud computing makes multiple systems on the Internet work by the interaction with virtual physical resources that conform the service architecture. In [1] Cloud computing is defined as Internet-based applications that can provide different information systems services including networking, filing and storage.

The innovation of using Cloud computing as a platform for the implementation of an information system inside an organization is due to the simplicity of configuring and programming the features that the providers offer [12], since by combining different hardware and software modalities in a virtual environment can increase the efficiency [4]. The authors in reference [1] highlight that cloud computing is the on-demand and expandable technology service offered over the Internet from data centers.

The next sections in this paper are organized in the following manner. In the second section the problem of the implementation of a CAT system in on-premises enviroments is explained. The third section deals with the solution proposal that involves the use of Cloud computing. The fourth section gives an introduction to CAT systems and its relationship with Cloud computing is explained. The fifth section shows and explains some screen test of the implementation of a prototype of CAT in Microsoft Azure Cloud platform. Finally, the sixth section provides some conclusions.

\section{The Problem}

Computer Adaptive Testing (CAT) is a technique that assumes the construction of items related to a previously given knowledge topic, which are then used to evaluate the abilities of a person in the aforementioned topic. This technique allows to finish the testing process in several ways and one of them consists in verifying that the most recent abilitie's values do not change within a given precision.

CAT systems based on IRT require a great computing capacity, since they are platforms that need high processing, availability and concurrence capacity. These needs arise from the fact that a proper CAT system requires the calibration of huge amount of items, which are related to a psychometric model containing a finite number of parameters, whose values depend on the corresponding item. In real scenarios, there does exist the possibility of handling at least one hundred items in a calibration process at a given instant of time, which means that one hundred parameters are required in the simplest case of the 1PL model or Rasch's psychometric model. In addition to this, and as a consequence of mobile devices development, CAT system must allow that the users access the testing service from any smart device, like cellphones or tablets, producing in this way the possibility of a huge demand on the part of the users and, as a consequence, a high concurrence of simultaneous access to the platform. 
Even worse, a CAT system considers three main actors or user types; namely, the administrators, the evaluators and the examinees. Furthermore, since the system can offer the service to different educational institutions, which can consider very diverse topics of any knowledge area, several students and evaluators can access the system over the Internet demanding a very robust hardware and software infrastructure for the correct function of the platform, and as a support for the future growing on the demand of the users.

Therefore, CAT systems pose the problem of satisfying the need of great power of processing while granting a high availability, a flexible growing storage, an acceptable bandwidth size to support the high concurrence and a management easiness in the whole resources for the good platform's performance. These needs are not easily obtained under the standard use of personal servers landed onpremises and some services offered by the Internet providers.

\section{Use of the Cloud Computing as an Alternative Solution}

As it has been written above, the implementation of a CAT system nowadays presents several challenges, mainly those related to the requirements of hardware and software introduced at the end of the previous section. For this to be successfully solved, this paper presents an alternative of solution through the use of a Cloud Computing infrastructure currently offered by Microsoft, which is called Azure. The reason of using this particular cloud computing provider is centered mainly on the author's knowledge about Microsoft's technologies; however, future work implies to make a deeper analysis about the features that other providers offer, in order to determine which one could fit the best for a CAT system implementation.

\section{Computer Adaptive Testing Systems and their Relationship with Cloud Computing}

CAT systems are platforms for testing some given knowledge areas commonly used in e-learning or Intelligent Tutoring systems (ITR) [5]. A CAT system has a very solid mathematical support tipically defined by the Item Response Theory (IRT), which contains the basis for the implementation of a calibration mechanism for the items that will conform the tests [9]. In other words, the system must initially contain a repository of calibrated items, which are fitted through a calibration algorithm previously selected. The algorithms for calibration require the specification of a psychometric model, which is defined in terms of parameters. In consequence, the calibration process searches for the proper values of the parameters related to every item, based on the selected psychometric model. The selection of the psychometric model depends on the scenario where the test is applied, and the system must give reliable information about the abilities of the examinee, the dificulty of the items and the test in general. 
When the values for the parameters are obtained, then these values can be useful in e-learning environments, Intelligent Tutoring Systems and CAT systems, which are examples of scenarios where a reliable and inmediate diagnostic is required to give support to the teaching-learning process.[7].

However, the implementation of a CAT system is not trivial since, as it has been aforementioned in this work, the system must completely cover the specific requirements to grant the correct operation of a platform of this kind. Nowadays, the digital revolution has transformed even the manner of teaching in the classroom and the testing of students, in such a way that a present-day testing mechanism is needed to bring both, the institutions and the students, to carry out these testing process by means of their digital devices. Internet is the more immediate alternative by making use of Cloud Computing service providers. In this way, a CAT system can be configured inside a platform that uses virtual physical resources, which interact in the infrastructure already hosted in a data center having the necessary support to host big amounts of informations systems, giving facilities for the access, configuration and management on the part of the users through a big bandwidth.

\subsection{The Cloud and the Virtual Machines}

Nowadays the cloud providers offer, as part of their services, the possibility of creating virtual machines with several combinations of resources that include memory sizes, hard disk storage capacity and the number of processor cores to be used. These benefits are important points to consider, when the creation of an online platform allowing the test of abilities in some knowledge topic is desired.

The considerations must include the high disponibility of the platform, which is granted by the right configuration of the virtual machine, the installation of an operating system in server version, the adequate assignment of the storage capacity, given that the system will have an extense number of items inside a repository and these items might contain hypermedia; in other words the items can include, in addition to plain text, embedded images, audio files, video or PDF documents. Then the necessary storage for all those items demands to the platform a big space in virtual hard disk.

On the other hand, it should carry out the right installation and configuration of the database manager, since it must create a database of non-calibrated items, a database of calibrated items, a database of testing results and a database with the information of the users and their access keys to the system. This represents an exponential growing in the databases due to the amount of users per educational institution having access to the platform.

Furthermore, there is the need of processing the CAT algorithms of the tests currently taken, applying the selected psychometric model and calculating the best item item choice along the test process. This leads to think in the concept of a real time system such as in the concurrency of the users, since in a same instant of time, the system could be testing a big amount of persons belonging to different educational institutions located in several geographic points [2], in different topics or knowledge areas, accessing from several types of digital and 
mobile devices. By being a platform online that is processing the answers for all the items in an intelligent manner, it requires a huge processing power, which tipically is expressed by the number of cores of the processor that can be assigned in the virtual machine.

If all these things will be implemented in only one physical server, like used to be so, then it will lead to the fast saturation of the capabilities and, in consequence, to the impossibility to bring a testing service of high availability with no possible scaling to cover the needs. Moreover, the considerations of publishing a system of this kind over the Internet must include the security topic, since the security of the already stored information should be granted [11][13], and the privacy of the registered users data should be kept, as well. Some of the concerns and effects occurring when the Cloud Computing is used as a host of a system with educational orientation can be find in some references [1].

\subsection{Microsoft Azure as a Cloud Computing Platform}

Azure is the cloud portal of Microsoft and offers very good options of services with a relative low cost in accordance to the needs that somebody may have. In other words, Azure allows to the users the creation of virtual machines, web sites, mobile services, among others, in a wide gamma of options that the management portal has. If advanced services to create Internet based systems are required, then Azure is one of the main platforms for Cloud Computing that can be used nowadays.

The present work precisely suggests how well the implementation of a prototype of adaptive testing system, in the cloud of Azure, allows the use of features of scalability that guarantee the correct operation of the platform, the high concurrency and enough space for data storage, which give an advice that this kind of implementations are an excellent alternative to get the computing capabilities that a CAT system really needs.

\subsection{Architecture of CAT System in the Cloud}

Some research results show an architecture of adaptive testing mounted over a web access platform [6]. However, the work uses the traditional structure consisting of a server in-house which, through a public IP over TCP/IP protocol, makes the system accessible from any device. The present work proposes a similar architecture, but inside the space offered by one Cloud service provider, which allows inmediate scalability, growing flexibility in the virtual physical resources on-demand, in addition to better bandwidths that can satisfy the high traffic and the user's concurrency.

The Fig. 1 shows the architecture that has been designed for the implementation of the Ariya Framework in the Cloud Computing service of Microsoft Azure. 


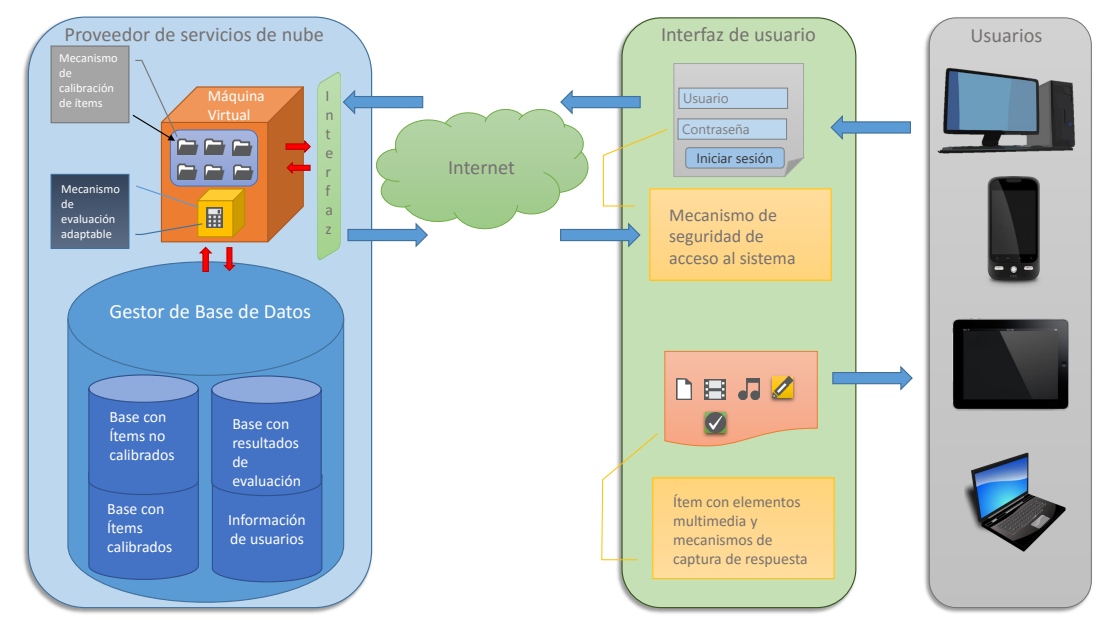

Fig. 1. Architecture diagram of the Framework Ariya in Azure

\section{Implementation of the Framework Ariya in the Azure's Cloud}

The implementation of the Framework Ariya in the cloud of Azure uses a Bizspark program consumer account to access the Azure management portal. Once inside the portal, the configuration of a virtual machine type A4 with 8 processor cores and 14GB of RAM memory is carried out. There is a fee for using the features of the cloud, but thinking in terms of the capabilities offered, like high processing and big RAM amount, the cost is worth to be payed. On the other hand, if one thinks for a while that the service will be given to different educational institutions to make their corresponding tests over this platform, it makes sense to think that the cost of the initial operation will be covered by the payed cuotes of rent for the Framework Ariya, as well.

With no doubt, the making of a business model to offer the services of the platform Ariya is necessary; however, the discussion about this component is left as a future work since the correct configuration of the prototype, and the making of the corresponding tests to garantee the right operation, should be made beforehand. The Fig. 2 shows the screencapture where the registration of a new cloud service for the Framework Ariya, inside the portal of Azure, appears.

\subsection{Configuration of the Virtual Environment in Azure for the Framework Ariya}

The Fig. 3 shows the screen capture of the virtual machine configured with the features previously described, relative to the Azure's management portal. The Fig. 4 shows the register of a virtual hard disk asociated to the platform Ariya with a Linux operating system. A version of Ubuntu server as the host for the 


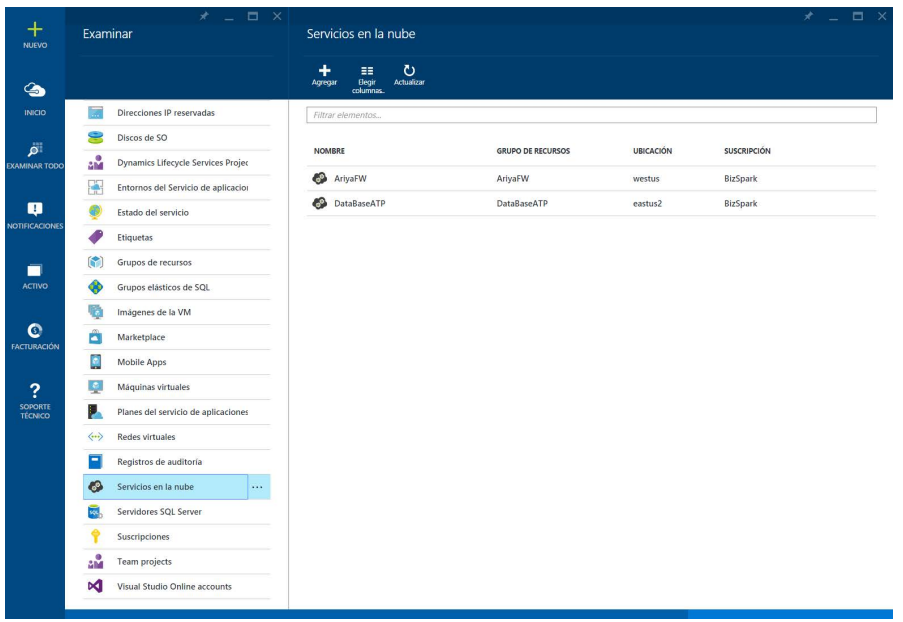

Fig. 2. Ariya cloud service in the portal of Azure

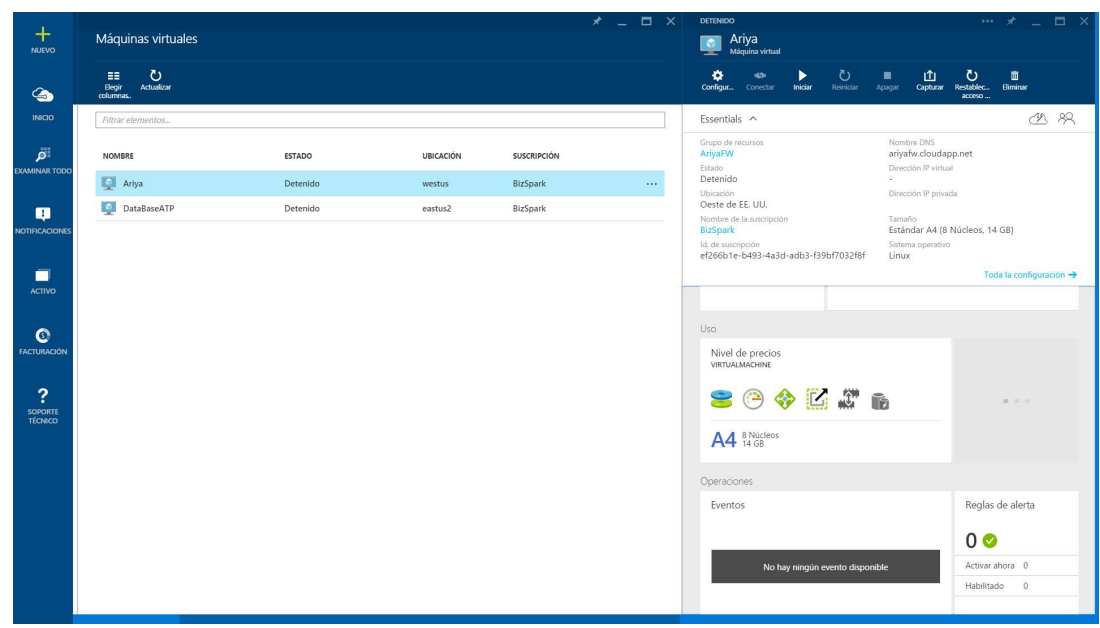

Fig. 3. Configuration of the virtual machine for the Framework Ariya in Azure 


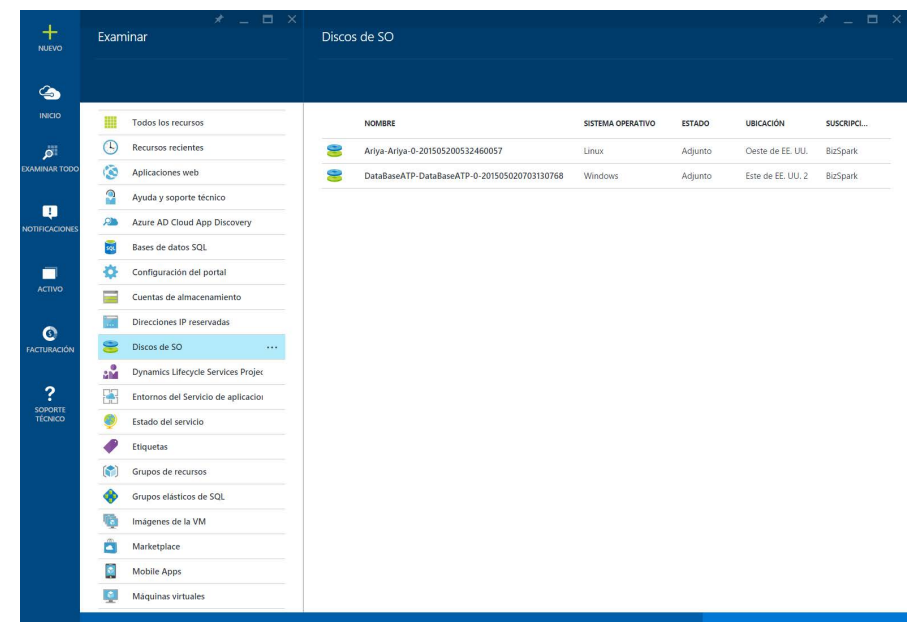

Fig. 4. Disk of the operating system assigned to Ariya's virtual machine

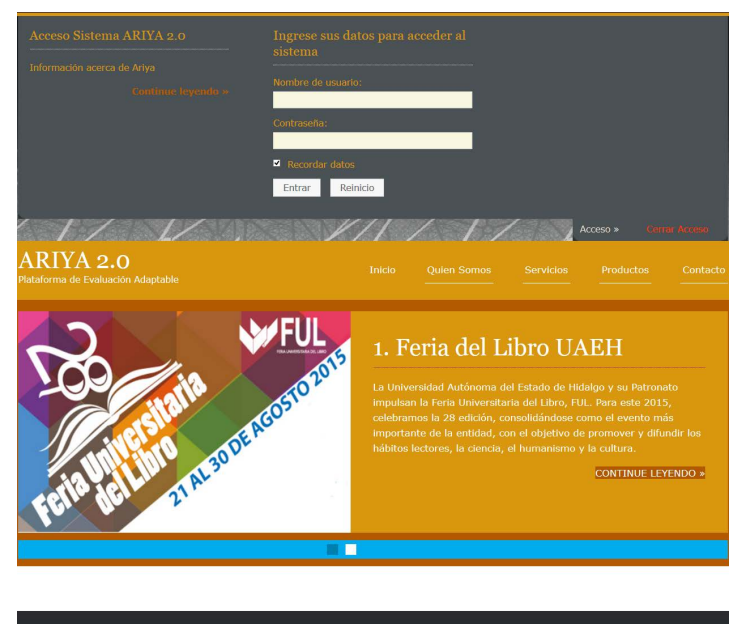

Fig. 5. Start user interface of the Framework Ariya 
CAT system has been configured in this implementation.

The start screen of the user interface for the Ariya system, ready to be accessed by a user through her/his user name and password, can be appreciated in the Fig. 5.

\section{Conclusions}

This work presents a proposal of implementation for a CAT system using the benefits that the Cloud Computing service providers offer nowadays. In particular, the use of the Microsoft Azure cloud service case is shown. The implementation of the Framework Ariya offers a pilot test of how this kind of adaptive testing systems could be worked in order to bring to the users the functionalities when this kind of platforms is used. The easiness for accessing the system from any digital or mobile smart device that the users can already own is among these functionalities, since this characteristic does not require the installation of any additional software, it is enough to have an Internet connection and to access from the web browser of the device.

The implementation of the CAT system in the Cloud allows the use of the great processing power of the multicore processors, from which the virtual machines can be configured, in addition to the sharing of storage space in the database and in the hard disk for the calibrated items, the richness of multimedia contents and the results of the tests. The adaptive testing mechanism will use the resources of processing power in order to calculate, almost in real time, the results of the adaptive testing and the items' calibration.

Furthermore, this paper discusses the implementation of a prototype of the Framework Ariya which intents to have all the elements of a fully operational CAT system, so that the adaptive evaluation tests using different psychometric models can be made in a future work, integrating inclusive more complex and resource demanding mathematical techniques [3].

\section{References}

1. Arpaci, I., Kilicer, K., Bardaki, S.: Effects of security and privacy concerns on educational use of cloud services. Computers in Human Behavior 45, 93-98 (2015)

2. Atabekova, A., Gorbatenko, R., Chilingaryan, K.: Student's attitude to cloud-based learning in university diverse environment: a case of rusia. Academic Journals 10(1), 1-9 (2014)

3. Cheng, S.C., Huang, Y.M., Chen, J.N., Lin, Y.T.: Automatic leveling system for e-learning examination pool using entropy-based decision tree. Lecture Notes in Computer Science pp. 273-278 (2005)

4. Grossman, R.: The case for cloud computing. IT Professional 11(2), 23-27 (2009)

5. Guzmn, E., Conejo, R.: A model for student knowledge diagnosis through adaptive testing. Lecture Notes in Computer Science 3220, 12 (2004)

6. Huang, Y., Lin, Y., Cheng, S.: An adaptive testing system for supporting versatile educational assessment. Computers and Education 52(1), 53-67 (2009) 
7. Lazarinis, F., Green, S., Pearson, E.: Creating personalized assessments based on learner knowledge and objectives in a hypermedia web testing application. Computers and Education 55, 1732-1743 (2010)

8. Lin, Y.T., Wen, M.L., Jou, M., Wu, D.W.: A cloud-based learning environment for developing student reflection abilities. Computers in Human Behavior 32(3), 244-252 (2014)

9. van der Linden, W., Hambleton, R.: Handbook of Modern Item Response Theory (1997)

10. Park, E., Kim, K.J.: An ntegrated adoption model of mobile cloud services: Exploration of key determinants and extension of technology acceptance model. Telematics and Informatics 31(3), 376-385 (2014)

11. Stantchev, V., Colomo-Palacios, R., Soto-Acosta, P., Misra, S.: Learning management systems and cloud file hosting services: A study on student's acceptance. Computers in Human Behavior 31, 612-619 (2014)

12. Sultan, N.: Making use of cloud computing for healthcare provision: Opportunities and challenges. International Journal of Information Management 34, 177-184 (2014)

13. Wang, C., Wang, Q., Ren, K., Cao, N., Lou., W.: Toward secure and dependable storage services in cloud computing. IEEE Transactions on Services Computing 5 (2), 220-232 (2012) 